\title{
Randomized Controlled Trial of Annual Zoledronic Acid to Prevent Gonadotropin-Releasing Hormone Agonist-Induced Bone Loss in Men With Prostate Cancer
}

\author{
M. Dror Michaelson, Donald S. Kaufman, Hang Lee, Francis J. McGovern, Philip W. \\ Kantoff, Mary Anne Fallon, Joel S. Finkelstein, and Matthew R. Smith \\ Division of Hematology and Oncology and Endocrine Unit, Department of Medicine, Department \\ of Urology, and Biostatistics Center, Massachusetts General Hospital; and the Department of \\ Adult Oncology, Dana-Farber Cancer Institute, Boston, MA.
}

\begin{abstract}
Purpose-Gonadotropin-releasing hormone $(\mathrm{GnRH})$ agonists decrease bone mineral density (BMD) and increase fracture risk in men with prostate cancer. Annual zoledronic acid increases BMD in postmenopausal women, but its efficacy in hypogonadal men is not known.

Patients and Methods-In a 12-month study, 40 men with nonmetastatic prostate cancer who were receiving a GnRH agonist and had $\mathrm{T}$ scores more than -2.5 were randomly assigned to zoledronic acid (4 mg intravenously on day 1 only) or placebo. BMD of the posteroanterior lumbar spine and proximal femur were measured by dual-energy x-ray absorptiometry.
\end{abstract}

Results-Mean $( \pm$ SE) BMD of the posteroanterior lumbar spine decreased by $3.1 \% \pm 1.0 \%$ in men assigned to placebo and increased by $4.0 \% \pm 1.0 \%$ in men assigned to zoledronic acid $(P<$. $001)$. BMD of the total hip decreased by $1.9 \% \pm 0.7 \%$ in men assigned to placebo and increased by $0.7 \% \pm 0.5 \%$ in men assigned to zoledronic acid $(P=.004)$. Similar between-group differences were observed for the femoral neck and trochanter. Serum N-telopeptide, a marker of osteoclast activity, decreased significantly after zoledronic acid treatment.

C 2007 by American Society of Clinical Oncology

Address reprint requests to Matthew R. Smith, MD, Massachusetts General Hospital, Department of Hematology/Oncology, 100 Blossom St, Cox 640, Boston, MA 02114; smith.matthew@mgh.harvard.edu.

\section{AUTHORS' DISCLOSURES OF POTENTIAL CONFLICTS OF INTEREST}

Although all authors completed the disclosure declaration, the following authors or their immediate family members indicated a financial interest. No conflict exists for drugs or devices used in a study if they are not being evaluated as part of the investigation. For a detailed description of the disclosure categories, or for more information about ASCO's conflict of interest policy, please refer to the Author Disclosure Declaration and the Disclosures of Potential Conflicts of Interest section in Information for Contributors. Employment: N/A Leadership: N/A Consultant: Matthew R. Smith, Novartis Oncology Stock: N/A Honoraria: M. Dror Michaelson, Novartis Oncology Research Funds: Matthew R. Smith, Novartis Oncology Testimony: N/A Other: N/A

AUTHOR CONTRIBUTIONS

Conception and design: M. Dror Michaelson, Donald S. Kaufman, Hang Lee, Francis J. McGovern, Philip W. Kantoff, Joel S. Finkelstein, Matthew R. Smith

Financial support: Matthew R. Smith

Administrative support: Mary Anne Fallon

Provision of study materials or patients: M. Dror Michaelson, Donald S. Kaufman, Francis J. McGovern, Philip W. Kantoff, Matthew R. Smith

Collection and assembly of data: M. Dror Michaelson, Hang Lee, Mary Anne Fallon, Matthew R. Smith

Data analysis and interpretation: M. Dror Michaelson, Hang Lee, Mary Anne Fallon, Joel S. Finkelstein, Matthew R. Smith Manuscript writing: M. Dror Michaelson, Philip W. Kantoff, Joel S. Finkelstein, Matthew R. Smith

Final approval of manuscript: M. Dror Michaelson, Donald S. Kaufman, Hang Lee, Francis J. McGovern, Philip W. Kantoff, Mary Anne Fallon, Joel S. Finkelstein, Matthew R. Smith 
Conclusion-In men receiving a GnRH agonist, a single treatment with zoledronic acid significantly increased BMD and durably suppressed serum N-telopeptide levels for 12 months. Annual zoledronic acid may be a convenient and effective strategy to prevent bone loss in hypogonadal men.

\section{INTRODUCTION}

A growing population of men with recurrent prostate cancer is treated with gonadotropinreleasing hormone $(\mathrm{GnRH})$ agonists. GnRH agonists are the mainstay of treatment for metastatic prostate cancer. In addition, GnRH agonists are a routine part of management for many men with either locally advanced or recurrent nonmetastatic prostate cancer. An estimated 600,000 men with prostate cancer are treated annually with GnRH agonists in the United States.

GnRH agonist therapy reduces serum testosterone to castrate levels. Men treated with GnRH agonists represent the largest population of hypogonadal men, and adverse consequences of hypogonadism are increasingly being recognized in these men. Well-described effects of hypogonadism include vasomotor flushing, loss of libido, fatigue, and anemia. More recent work has demonstrated that osteoporosis is an important complication of GnRH agonist treatment in men with prostate cancer. GnRH agonists increase bone turnover, ${ }^{1}$ decrease bone mineral density (BMD), ${ }^{1-} 4$ and increase fracture risk. $5^{-7}$

In a randomized, placebo-controlled study of men with nonmetastatic prostate cancer receiving GnRH agonists for prostate cancer, zoledronic acid (4 mg intravenously every 3 months) significantly increased BMD. ${ }^{8}$ In postmenopausal women with osteoporosis, less frequent treatment with zoledronic acid (4 mg intravenously annually) significantly increased BMD and durably suppressed biochemical markers of osteoclast activity compared with placebo. 9 The efficacy of annual zoledronic acid in hypogonadal men is unknown.

We conducted a randomized, placebo-controlled study to evaluate the efficacy of annual zoledronic acid in men receiving GnRH agonists for prostate cancer. The main study outcomes were changes in BMD and biochemical markers of bone turnover from baseline to month 12 .

\section{PATIENTS AND METHODS}

\section{Patients}

Study participants were recruited at Massachusetts General Hospital and Dana-Farber Cancer Institute between September 2003 and March 2005. All patients had prostate adenocarcinoma and were receiving treatment with a GnRH agonist at study entry. Patients had a radionuclide bone scan within 6 months before initiating GnRH agonist treatment or 6 months before study entry. Men with bone metastases or evidence of progressive disease (serum prostate-specific antigen $>150 \%$ nadir value) were excluded. Men with metabolic bone disease, history of treatment for osteoporosis, history of deep venous thrombosis or pulmonary embolus, serumcalcium less than $8.4 \mathrm{mg} / \mathrm{dL}$ or more than $10.6 \mathrm{mg} / \mathrm{dL}$, or serum creatinine concentration more than $2.0 \mathrm{mg} / \mathrm{dL}(177 \mu \mathrm{mol} / \mathrm{L})$ were also excluded. Other exclusion criteria included chronic use of glucocorticoids, anticonvulsants, or suppressive doses of thyroxine within 1 year or any treatment with bisphosphonates within 1 year.

At a screening visit, BMD of the posteroanterior lumbar spine and proximal femur was determined by dual-energy $\mathrm{x}$-ray absorptiometry. Patients with a $\mathrm{T}$ score $\leq-2.5$ were excluded. 


\section{Study Design}

After a screening visit, eligible patients were randomly assigned using computer-generated cards to receive zoledronic acid $4 \mathrm{mg}$ (Zometa; Novartis Pharmaceuticals Inc, Basel, Switzerland) or placebo intravenously on day 1 only. All patients and study personnel were blinded to treatment assignments. Patients in both groups continued treatment with a GnRH agonist throughout the study. All patients received calcium carbonate (500 mg daily) and a daily multivitamin containing vitamin D (400 U).

Patients were evaluated at baseline and at 3, 6, 9, and 12 months. A serum sample was obtained at each visit and stored at $-80^{\circ} \mathrm{C}$. BMD and body composition were measured by dual-energy x-ray absorptiometry at baseline and 12 months. The institutional review board approved the study. All patients provided written informed consent.

\section{Study End Points}

BMD of the posteroanterior lumbar spine and proximal femur was determined by dualenergy x-ray absorptiometry using a Hologic QDR 4500A densitometer (Hologic Inc, Waltham, MA). Our short-term in vivo measurement standard deviations (with repositioning) are $0.005,0.007$, and $0.006 \mathrm{~g} / \mathrm{cm}^{2}$ for posteroanterior spine, femoral neck, and total hip, respectively. Serum concentrations of testosterone (Diagnostic Products, Los Angeles, CA) were measured by radioimmunoassays. Serum concentrations of Ntelopeptide (Ostex International, Seattle, WA) and bone alkaline phosphatase (Metra Biosystems, Mountain View, CA) were measured by enzyme immunoassays.

\section{Statistical Analyses}

The primary study end point was the percent change in the BMD of the posteroanterior lumbar spine from baseline to month 12 . The power calculations for this study assumed a $3.3 \%$ standard deviation of the change from baseline ${ }^{1}$ and dropout rate of $15 \%$. The sample size of 40 patients (20 patients per group) provided $80 \%$ power to detect a difference of at least 3.3\% using a two-sided $t$ test $(\alpha=.05)$. Percent changes in BMD, testosterone, Ntelopeptide, and bone alkaline phosphatase were compared between groups using $t$ tests. All data were included in the efficacy analyses.

Statistical analyses were performed using SAS Version 8.1 (SAS Institute, Cary, NC). Values are reported as means \pm SE unless specified otherwise. All $P$ values are two sided, and $P<.05$ is considered statistically significant.

\section{RESULTS}

\section{Characteristics of the Patients}

Forty-four eligible patients were randomly assigned to either zoledronic acid $(\mathrm{n}=22)$ or placebo $(n=22)$. Baseline characteristics of men in both groups were similar (Table 1). All patients were receiving treatment with a GnRH agonist at study entry and continued GnRH agonist therapy throughout the 12-month study period. At baseline, duration of prior $\mathrm{GnRH}$ agonist treatment was $12 \pm 16$ months for men assigned to zoledronic acid and $21 \pm 17$ months for men assigned to placebo. Thirty-six patients completed BMD testing at 12 months.

\section{BMD}

Mean percent changes in BMD of the posteroanterior lumbar spine differed significantly between groups (Table 2). Mean ( \pm SE) BMD of the posteroanterior lumbar spine decreased by $3.1 \% \pm 1.0 \%$ in the placebo group from baseline to 12 months. In contrast, BMD 
increased by $4.0 \% \pm 1.0 \%$ in the zoledronic acid group $(P<.001)$. The between-group difference in percent change from baseline to 12 months was $7.1 \%(95 \% \mathrm{CI}, 4.2 \%$ to $10.0 \%)$

Mean percent changes in BMD of the total hip and trochanter also differed significantly between groups (Table 2). BMD of the total hip decreased by $1.9 \% \pm 0.7 \%$ in the placebo group and increased by $0.7 \% \pm 0.5 \%$ in the zoledronic acid group $(P=.004)$. BMD of the trochanter decreased by $1.4 \% \pm 0.7 \%$ in the placebo group and increased by $1.7 \% \pm 0.8 \%$ in the zoledronic acid group $(P=.008)$. The between-group differences in percent change from baseline to 12 months were $2.6 \%$ (95\% CI, $0.8 \%$ to $4.2 \%$ ) for the total hip, $3.1 \%$ (95\% CI, $0.9 \%$ to $5.3 \%$ ) for the trochanter, and $4.4 \%$ (95\% CI, $-0.1 \%$ to $4.4 \%$ ) for the femoral neck.

\section{Biochemical Markers of Bone Turnover}

Changes from baseline to 12 months in serum $\mathrm{N}$-telopeptide and bone specific alkaline phosphatase concentrations differed significantly between the groups (Fig 1). Mean ( \pm SE) serum N-telopeptide increased by $10 \% \pm 8 \%$ in the placebo group and decreased by $17 \% \pm$ $6 \%$ in the zoledronic acid group $(P=.01)$. Serum bone-specific alkaline phosphatase increased by $15 \% \pm 8 \%$ in the placebo group and decreased by $13 \% \pm 4 \%$ in the zoledronic acid group $(P=.003)$. The between-group difference in percent change from baseline to 12 months was $27 \%$ (95\% CI, $6 \%$ to $48 \%$ ) for N-telopeptide and $27 \%$ (95\% CI, $11 \%$ to $44 \%$ ) for bone-specific alkaline phosphatase.

Zoledronic acid seemed to achieve durable suppression of bone turnover. In the zoledronic acid group, mean serum $\mathrm{N}$-telopeptide and bone-specific alkaline phosphatase concentrations decreased sharply from baseline to month 3 and remained stable from month 3 to month 12. There was no significant difference between month 3 and month 12 in either $\mathrm{N}$-telopeptide or bone-specific alkaline phosphatase in men treated with zoledronic acid.

\section{Adverse Events}

There were no serious adverse events related to treatment in either group.

\section{DISCUSSION}

This study demonstrated that a single infusion of zoledronic acid suppressed bone turnover for at least 12 months and increased BMD of the hip and spine in men receiving a GnRH agonist for nonmetastatic prostate cancer. Compared with placebo, zoledronic acid increased BMD of the lumbar spine and hip by $7.1 \%$ and $2.6 \%$, respectively. In a placebo-controlled study of 106 men with nonmetastatic prostate cancer, zoledronic acid administered every 3 months increased BMD of the lumbar spine and hip by $7.8 \%$ and $3.9 \%$, respectively. ${ }^{8}$ Although the current study did not directly compare the two schedules of zoledronic acid treatment, the similarity of BMD results and persistent suppression of serum $\mathrm{N}$-telopeptide throughout the 12-month study suggest that annual zoledronic acid is sufficient to prevent bone loss in hypogonadal men.

The observed efficacy of annual zoledronic acid in these hypogonadal men with prostate cancer is consistent with the results of zoledronic acid in postmenopausal women with osteoporosis. In a multicenter, randomized trial, 351 postmenopausal women were assigned to receive placebo or zoledronic acid every 3,6 , or 12 months, and effects on BMD and biochemical markers of bone turnover were studied. ${ }^{9}$ In women treated with once-yearly zoledronic acid, changes in spine and femoral neck BMD were significantly higher than in women treated with placebo at 12 months after treatment, and bone resorption markers remained suppressed after 12 months. The changes seen with annual zoledronic acid were 
comparable to changes seen with more frequent administration. The study concluded that annual infusion of zoledronic acid is effective therapy for postmenopausal osteoporosis.

Treatment with bisphosphonates can have adverse consequences. An emerging literature has linked bisphosphonates, particularly high doses of zoledronic acid, with osteonecrosis of the jaw. ${ }^{10-13}$ Zoledronic acid is also associated with a risk of renal failure when administered in doses much higher than in the current study. ${ }^{14}$ The relative risk of osteonecrosis, and likely of other potential complications, may increase with the cumulative dose of zoledronic acid. Thus, if comparable efficacy can be achieved with less frequent administration, fewer adverse consequences may be observed.

Other bisphosphonates also increase BMD in GnRH agonist-treated men. A randomized, placebo-controlled trial of 47 men with prostate cancer demonstrated that pamidronate (60 mg intravenously every 3 months) prevents loss of BMD in the hip and spine in men initiating treatment with $\mathrm{GnRH}$ agonists. ${ }^{1} \mathrm{BMD}$ of the spine, trochanter, and hip significantly declined $(3.3 \% \pm 0.7 \%, 2.1 \% \pm 0.6 \%$, and $1.8 \% \pm 0.4 \%$, respectively) in men treated with $\mathrm{GnRH}$ agonists and placebo but did not significantly change at any skeletal site in men treated with GnRH agonists and pamidronate. Similar results were reported in a second randomized controlled trial, in which men treated with pamidronate manifested increased BMD of the spine and femoral neck compared with men treated with placebo. 15 In both studies, pamidronate significantly reduced levels of bone turnover markers compared with placebo.

GnRH agonist-induced bone loss can also be prevented with selective estrogen receptor modulators such as raloxifene and toremifene in men with prostate cancer.16,17 Other agents, including denosumab (AMG-162; Amgen, Thousand Oaks, CA), a novel antibody targeted against receptor activator of nuclear factor-kb ligand, are currently being investigated as potential therapies for osteoporosis in men with nonmetastatic prostate cancer.

In men with androgen-independent prostate cancer and bone metastases, frequent treatment with zoledronic acid (4 mg every 3 weeks) decreases the risk of disease-related skeletal complications, including fractures. ${ }^{18}$ In a double-blind randomized trial of 643 men with androgen-independent prostate cancer, zoledronic acid (4 or $8 \mathrm{mg}$ every 3 weeks) reduced the relative risk of developing skeletal-related events (defined as pathologic bone fractures, spinal cord compression, surgery to bone, radiation therapy to bone, or a change of antineoplastic therapy to treat bone pain) by $25 \%$ and increased the median time to first skeletal-related event by more than 160 days. Given the results of this study, zoledronic acid ( $4 \mathrm{mg}$ every 3 to 4 weeks) was approved to treat men with hormone-refractory prostate cancer metastatic to bone, and this remains the only known effective schedule to prevent disease-related skeletal complications in men with metastatic prostate cancer. Our observation that annual zoledronic acid increases BMD in men with nonmetastatic prostate cancer does not justify less frequent administration to prevent disease-related skeletal complications in men with metastatic prostate cancer.

There are some potential limitations to this study. The study was powered to detect a significant change in BMD and was not powered to assess the impact on fracture risk. Larger randomized trials would be required to assess the effect of different schedules of zoledronic acid on fracture risk. Also, only a single schedule of zoledronic acid administration was analyzed; additional studies are needed to determine whether even less frequent administration might provide similar benefits. The baseline characteristics of both groups were similar, although the mean duration of prior $\mathrm{GnRH}$ agonist treatment was longer in men assigned to zoledronic acid than in men assigned to placebo. Because BMD 
decreases at a steady rate regardless of the duration of past GnRH agonist exposure, ${ }^{19}$ this difference would not seem to explain the observed significant differences in changes in BMD and biochemical markers between the groups. Finally, the patient population in this study was restricted to nonmetastatic prostate cancer, and extrapolation to men with bone metastases would not be appropriate.

In summary, zoledronic acid (4 mg once in 12 months) significantly increased BMD of the hip and spine in men receiving a GnRH agonist for prostate cancer. Annual zoledronic acid may represent a convenient and effective strategy to prevent osteoporosis in hypogonadal men.

\section{Acknowledgments}

We thank the dedicated staff of the Massachusetts General Hospital Bone Density Center.

Supported by the W. Bradford Ingalls Foundation and research awards from the Prostate Cancer Foundation and Novartis Oncology.

The study sponsors played no role in the study design; in collection, analysis, and interpretation of data; or in writing of this report.

\section{REFERENCES}

1. Smith MR, McGovern FJ, Zietman AL, et al. Pamidronate to prevent bone loss during androgendeprivation therapy for prostate cancer. N Engl J Med 2001;345:948-955. [PubMed: 11575286]

2. Maillefert JF, Sibilia J, Michel F, et al. Bone mineral density in men treated with synthetic gonadotropin-releasing hormone agonists for prostatic carcinoma. J Urol 1999;161:1219-1222. [PubMed: 10081873]

3. Diamond T, Campbell J, Bryant C, et al. The effect of combined androgen blockade on bone turnover and bone mineral densities in men treated for prostate carcinoma: Longitudinal evaluation and response to intermittent cyclic etidronate therapy. Cancer 1998;83:1561-1566. [PubMed: 9781950]

4. Eriksson S, Eriksson A, Stege R, et al. Bone mineral density in patients with prostatic cancer treated with orchidectomy and with estrogens. Calcif Tissue Int 1995;57:97-99. [PubMed: 7584882]

5. Shahinian VB, Kuo YF, Freeman JL, et al. Risk of fracture after androgen deprivation for prostate cancer. N Engl J Med 2005;352:154-164. [PubMed: 15647578]

6. Smith MR, Lee WC, Brandman J, et al. Gonadotropin-releasing hormone agonists and fracture risk: A claims-based cohort study of men with nonmetastatic prostate cancer. J Clin Oncol 2005;23:7897-7903. [PubMed: 16258089]

7. Smith MR, Boyce SP, Moyneur E, et al. Risk of clinical fractures after gonadotropin-releasing hormone agonist therapy for prostate cancer. J Urol 2006;175:136-139. [PubMed: 16406890]

8. Smith MR, Eastham J, Gleason DM, et al. Randomized controlled trial of zoledronic acid to prevent bone loss in men receiving androgen deprivation therapy for nonmetastatic prostate cancer. J Urol 2003;169:2008-2012. [PubMed: 12771706]

9. Reid IR, Brown JP, Burckhardt P, et al. Intravenous zoledronic acid in postmenopausal women with low bone mineral density. N Engl J Med 2002;346:653-661. [PubMed: 11870242]

10. Tarassoff P, Csermak K. Avascular necrosis of the jaws: Risk factors in metastatic cancer patients. J Oral Maxillofac Surg 2003;61:1238-1239. [PubMed: 14586868]

11. Marx RE, Sawatari Y, Fortin M, et al. Bisphosphonate-induced exposed bone (osteonecrosis/ osteopetrosis) of the jaws: Risk factors, recognition, prevention, and treatment. J Oral Maxillofac Surg 2005;63:1567-1575. [PubMed: 16243172]

12. Bamias A, Kastritis E, Bamia C, et al. Osteonecrosis of the jaw in cancer after treatment with bisphosphonates: Incidence and risk factors. J Clin Oncol 2005;23:8580-8587. [PubMed: 16314620] 
13. Woo SB, Hellstein JW, Kalmar JR. Systematic review: Bisphosphonates and osteonecrosis of the jaws. Ann Intern Med 2006;144:753-761. [PubMed: 16702591]

14. Chang JT, Green L, Beitz J. Renal failure with the use of zoledronic acid. N Engl J Med 2003;349:1676-1679. [PubMed: 14573746]

15. Diamond TH, Winters J, Smith A, et al. The antiosteoporotic efficacy of intravenous pamidronate in men with prostate carcinoma receiving combined androgen blockade: A double blind, randomized, placebo-controlled crossover study. Cancer 2001;92:1444-1450. [PubMed: 11745221]

16. Smith MR, Fallon MA, Lee H, et al. Raloxifene to prevent gonadotropin-releasing hormone agonist-induced bone loss in men with prostate cancer: A randomized controlled trial. J Clin Endocrinol Metab 2004;89:3841-3846. [PubMed: 15292315]

17. Taneja SS, Smith MR, Dalton JT, et al. Toremifene: A promising therapy for the prevention of prostate cancer and complications of androgen deprivation therapy. Expert Opin Investig Drugs 2006;15:293-305.

18. Saad F, Gleason DM, Murray R, et al. A randomized, placebo-controlled trial of zoledronic acid in patients with hormone-refractory metastatic prostate carcinoma. J Natl Cancer Inst 2002;94:14581468. [PubMed: 12359855]

19. Lee H, McGovern K, Finkelstein JS, et al. Changes in bone mineral density and body composition during initial and long-term gonadotropin-releasing hormone agonist treatment for prostate carcinoma. Cancer 2005;104:1633-1637. [PubMed: 16116596] 


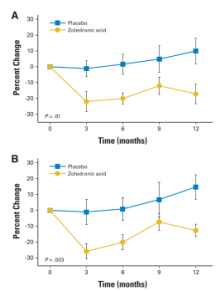

Fig 1.

Mean ( \pm SE) changes from baseline for (A) serum N-telopeptide and (B) serum bone alkaline phosphatase. $P$ values are for between-group comparisons of the percent change from baseline to month 12 . 
$\underset{\frac{0}{0}}{\frac{0}{0}}$

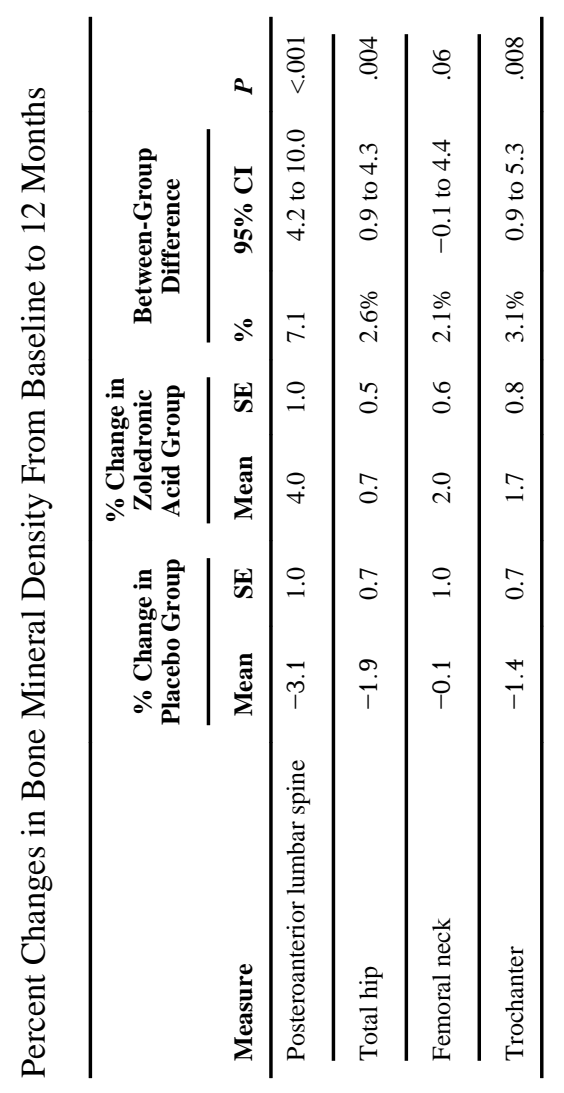

